\title{
P I5-05. Evaluation and recommendations on good clinical laboratory practice (GCLP) guidelines for phase I-III HIV vaccine clinical trials
}

\author{
M Sarzotti-Kelsoe*1,2, J Cox ${ }^{9}$, N Cleland ${ }^{3}$, T Denny ${ }^{4}$, J Hural ${ }^{5}$, L Needham ${ }^{4}$, \\ D Ozaki $^{6}$, IR Rodriguez-Chavez ${ }^{7}$, G Stevens ${ }^{9}$, T Stiles ${ }^{8}$, T Tarragona-Fiol $^{9}$ and
} A Simkins ${ }^{10}$

\begin{abstract}
Address: ${ }^{1}$ Immunology and Surgery, Duke University Medical Center, Durham, NC, USA, ${ }^{2}$ International AIDS Vaccine Initiative, Rockville, MD, USA, ${ }^{3}$ Henry M. Jackson Found. Advancement Mil. Medicine, DAIDS, NIAID, NIH, Bethesda, MD, USA, ${ }^{4}$ Duke Human Vaccine Institute, CHAVI, Durham, NC, USA, ${ }^{5}$ HIV Vaccine Trials Network, Seattle, WA, USA, ${ }^{6}$ CAVD/CA-VIMC Central QAU, Durham, NC, USA, ${ }^{7}$ AIDS and Immunosuppression Program, IBIDB, DER, NIDCR, NIH, Bethesda, MD, USA, ${ }^{8}$ Qualogy Ltd., Kettering, United Kingdom (Great Britain), ${ }^{9}$ International AIDS Vaccine Initiative Core Lab., Imperial College, London, United Kingdom (Great Britain) and ${ }^{10} \mathrm{AlphaVax}$ Human Vaccines, Inc., Research Triangle Park, USA

* Corresponding author
\end{abstract}

from AIDS Vaccine 2009

Paris, France. 19-22 October 2009

Published: 22 October 2009

Retrovirology 2009, 6(Suppl 3):P206 doi:I0.1 I86/1742-4690-6-S3-P206

This abstract is available from: http://www.retrovirology.com/content/6/S3/P206

(C) 2009 Sarzotti-Kelsoe et al; licensee BioMed Central Ltd.

\section{Background}

The Global HIV Vaccine Enterprise created a worldwide alliance of independent organizations dedicated to the development of preventive HIV vaccines. Global clinical laboratory work performed under harmonized operations is a central component for the successful conduct of phase I-III HIV vaccine clinical trials. In an effort to harmonize international laboratory operations, the British Association of Research Quality Assurance produced GCLP guidelines. Subsequently, the Division of AIDS, National Institute of Allergy and Infectious Diseases, National Institutes of Health expanded the existing knowledge on GCLP standards by publishing guidelines on GCLP with increased implementation guidance. Although both of these GCLP approaches ensure that laboratory results from multiple clinical laboratories conducting safety, diagnostic and endpoint assays are reliable, repeatable, auditable, and comparable, they differ in the interpretation of four critical GCLP elements: training, auditing/ accreditation, assay validation, and proficiency testing. These elements represent crucial stages in the conduct of GCLP-compliant studies performed by clinical laboratories: from the general set-up (training of personnel and assay validation) through assay conduction and laboratory oversight (audits, proficiency testing and accreditation).

\section{Methods}

A workshop was organized to bring together for the first time experts on GCLP to review, discuss the four elements listed above and harmonize the two main GCLP approaches into a single set of recommendations that can be followed by laboratories in the HIV field and in other scientific and medical.

\section{Conclusion}

The authors reached general consensus on three out of the four GCLP elements described above, except for the issue of GCLP laboratory accreditation. The latter is essential for GCLP compliance and remains controversial as currently, there is not a public internationally available accrediting organization acceptable to all parties involved in clinical trials. The authors wish to raise awareness on the issue of GCLP accreditation and the need to establish a global accrediting body. 